\title{
Binary pseudo-random gratings and arrays for calibration of the modulation transfer function of surface profilometers: recent developments
}

\author{
Samuel K. Barber, ${ }^{* a}$ Paul Soldate, ${ }^{b}$ Erik H. Anderson, ${ }^{a}$ Rossana Cambie, ${ }^{a}$ Stefano Marchesini, ${ }^{a}$ \\ Wayne R. McKinney, ${ }^{\mathrm{a}}$ Peter Z. Takacs, ${ }^{\mathrm{c}}$ Dmitriy L. Voronov, ${ }^{\mathrm{a}}$ Valeriy V. Yashchuk ${ }^{\mathrm{a}}$ \\ ${ }^{a}$ Lawrence Berkeley National Laboratory, Berkeley, California, 94720 \\ ${ }^{\mathrm{b}}$ Rensselaer Polytechnic Institute, Troy, New York, 12180 \\ ${ }^{c}$ Brookhaven National Laboratory, Upton, New York, 11973
}

\begin{abstract}
The major problem of measurement of a power spectral density (PSD) distribution of the surface heights with surface profilometers arises due to the unknown Modulation Transfer Function (MTF) of the instruments. The MTF tends to distort the PSD at higher spatial frequencies. It has been suggested [Proc. SPIE 7077-7, (2007), Opt. Eng. 47 (7), 073602-1-5 (2008)] that the instrumental MTF of a surface profiler can be precisely measured using standard test surfaces based on binary pseudo-random (BPR) patterns. In the cited work, a one dimensional (1D) realization of the suggested method based on use of BPR gratings has been demonstrated. Here, we present recent achievements made in fabricating and using two-dimensional (2D) BPR arrays that allow for a direct 2D calibration of the instrumental MTF. The 2D BPRAs were used as standard test surfaces for 2D MTF calibration of the Micromap ${ }^{\mathrm{TM}}{ }_{-} 570$ interferometric microscope with all available objectives. The effects of fabrication imperfections on the efficiency of calibration are also discussed.
\end{abstract}

Keywords: surface metrology, surface profilometer, interferometric microscope, modulation transfer function, MTF, power spectral density, PSD, calibration, error reduction, fabrication tolerances, metrology of $\mathrm{x}$-ray optics

\section{INTRODUCTION}

Roughness and figure specifications for state-of-the-art x-ray optics consistently push the limits of surface profilometry. In order to keep pace with growing demands of sub-Angstrom surface roughnesses and sub-micro radian slope variations, new surface profilometers and measurement techniques must be developed in parallel that achieve the desired precision. ${ }^{1,2}$ Addressing systematic errors unique to each instrumental system is a critical step in realizing this goal. A common systematic error of a surface profilometer is the unknown impulse response, or point spread function (PSF), inherent to an instrumental setup which tends to distort measurements. The PSF is interpreted as an irradiance distribution as a function of position that convolves with an ideal image to yield a measured image. Contributions to the PSF come from various sources including, but not limited to, the instrument's optical system, detector, signal processing and software algorithm. In principle it is possible to measure or calculate the PSF of each component separately and combine them to determine the entire system's PSF. ${ }^{3}$

However, when correcting these distortions it is often more convenient to work in the spatial frequency domain rather than the spatial domain for several reasons. Firstly, a convolution in the spatial domain is equivalent to a multiplication in the spatial frequency domain, which is far simpler to work with. In this domain the PSF is referred to the optical transfer function (OTF) which is generally a complex function comprised of a magnitude and phase portion called the modulation transfer function (MTF) and phase transfer function (PTF), respectively. ${ }^{3}$ Additionally, the power spectral density (PSD) distribution of a surface under test (SUT), obtained by the square of a straightforward Fourier transform of the measured height distribution, provides a basis for a more rigorous statistical description of the surface topography than a single value obtained from roughness calculations. ${ }^{4-7}$ For example, the measured PSD distributions provide a closed set of data necessary for three-dimensional calculations of scattering of light by optical surfaces. ${ }^{8-10}$ When squared, the phase portion of the OTF drops out which provides the following relation:

$$
P S D_{\text {measured }}=P S D_{S U T} \times M T F^{2}
$$


The MTF in Eq. (1) is the total MTF of the instrumental system, and is a product of the individual components. As such, it provides a simple method to experimentally determine the MTF of an entire instrumental system. The MTF can be determined by comparing the measured PSD distribution of a known test surface to the ideal numerically simulated PSD distribution of the same SUT. The square root of the ratio of the measured PSD distribution to the simulated PSD distribution gives the MTF of the system.

The effectiveness of this method hinges critically on the appropriate choice of test surface. Some common test patterns used in MTF measurements include bar targets, ${ }^{11}$ knife-edge sources, ${ }^{12,13}$ (step height standard ${ }^{14}$ ) and white noise patterns. ${ }^{15}$ In Refs.[16,17] a new type of test surface based on binary pseudo random (BPR) patterns, gratings and arrays that are specifically designed for MTF measurement of surface profilers, has been suggested as use as a calibration standard. The effectiveness of the method was demonstrated experimentally with a BPR grating which is a 1D realization of the method. The BPR surfaces have numerous advantages over other surfaces. The major distinguishing properties are equal sensitivity to the entire spatial frequency range of an instrument and deterministic character of the inherent PSD ${ }^{16,17}$.

In the present work, we expand upon the realization using 1D BPRGs and discuss binary pseudo-random arrays (BRPA), which are suitable for the direct measurement of the 2D MTF, and present a comprehensive analysis on the influence of fabrication imperfections that may affect MTF measurement. We show that the fabrication imperfection leads to only a $10-15 \%$ perturbation of the inherent PSD of a BPRA. Analytical methods for correction of the inherent BPRA PSD are developed and used to measure the MTF of the Micromap ${ }^{\mathrm{TM}}-570$ interferometric microscope.

\section{BINARY PSEUDO-RANDOM SEQUENCES AND ARRAYS AS TEST SURFACES}

\subsection{Background}

Binary arrays play an important role in astronomy, where they are employed as imaging detectors for x- and gamma-ray sources. After their original conception in the late $60 \mathrm{~s},{ }^{18,19}$ different aperture designs were proposed and evaluated for their imaging capabilities. An important advance was made in $1978^{20}$ with the introduction of the uniformly redundant array (URA), a design possessing both high throughput (50\%) and flat PSD spectrum (when sampled at the Nyquist frequency). Tracking the mentioned similarity of the binary pseudo-random sequences and uniformly redundant arrays, we employ terms BPRG and BPRA rather than URA for the latter one, when discussing the test surfaces used for the MTF calibration.

Today the URA remains the aperture of choice for many applications, including medical imaging, plasma research, homeland security, and x-ray holography. The URA's superior imaging capability originates from the fact that its cyclical autocorrelation function is a delta function. URA's are related by the fact that they can be constructed from pseudo-random (PR) sequences. Although we have chosen the term 'pseudo-random sequence', such sequences are alternatively referred to as pseudo noise sequences or m-sequences. ${ }^{21}$ A PR sequence is a special type of binary sequence with a two-valued periodic autocorrelation function. It has been extensively studied in the literature. ${ }^{22}$ What distinguishes one particular URA pattern from any other is the specific PR sequence chosen and the details of the packing used to transform the linear array of binary numbers into a 2-D pattern. One-dimensional URAs also can be used. The URA used in this paper follows the original prescription of the twin-prime class. In its original form, the URA was configured as a rectangular aperture of dimensions $r \times s$, where $r$ and $s$ are prime numbers and $r=s+2$. Thus, the matrix $A(i, j)=A(I, J)$, where $I=\bmod _{r} i$ and $J=\bmod _{s} j$. Furthermore, ${ }^{20}$

$$
\begin{aligned}
A(I, J) & =0 \quad \text { if } \quad I=0 \\
& =1 \quad \text { if } \quad J=0, I \neq 0 \\
& =1 \quad \text { if } \quad C_{r}(I) C_{s}(J)=1 \\
& =0 \quad \text { otherwise. }
\end{aligned}
$$

where

$$
\begin{aligned}
C_{r}(I)=1 & \text { if thereexists an integer } x, 1<x<r \\
& \text { suchthat } I=\bmod _{r} x^{2} \\
=-1 & \text { otherwise. }
\end{aligned}
$$


For more details see Ref. [20].

\subsection{Use as MTF calibration standard}

Using test surfaces based on BPR sequences and arrays has a number of advantages compared to test surfaces based on other commonly used patterns ${ }^{16,17}$ used for MTF measurements such as bar targets, knife edge sources, and white noise sources. A single bar pattern can only be used for measuring the MTF at a single fundamental spatial frequency at a time. Moreover, for each fundamental frequency, a series of measurements at harmonic frequencies need to be made to determine the MTF at the fundamental frequency. Consequently, obtaining the MTF over a range of spatial frequencies can be a very long and tedious process requiring numerous measurements. ${ }^{3,11}$

In surface profilometry a knife edge source can be modeled as a step height artifact. The use of such a surface for MTF measurement of surface profilers has been presented in Ref. [14]. There are a number of disadvantages in using these surfaces. The inverse quadratic dependence of the PSD spectrum on spatial frequency limits the sensitivity to the higher spatial frequency distortion. It also proves necessary to preprocess the data to filter out PSD variations at higher spatial frequencies. Furthermore, the MTF calibration exhibits a strong dependence on the position of the step height within the field of view of the instrument.

The advantage of white noise sources is that the inherent PSD should be essentially flat which provides equal sensitivity to the instrumental MTF over the entire spatial frequency range. Any deviation in the measured PSD distribution from a flat PSD distribution is a direct measure of the instrumental MTF. From a practical standpoint, however, developing and using test surfaces based on generic white noise sources for MTF measurement of profiling instruments can be problematic.

The primary property of BPR sequences and arrays that makes them an attractive option for MTF measurement test surfaces is their inherently flat (i.e. spatial frequency independent) PSD distributions. In contrast to a white noise source, this property is guaranteed by the deterministic spacing of the binary elements such that the cyclic autocorrelation function is a delta function. Therefore, BPR test surfaces are easy to specify for standard micro- and nano-fabrication processes. For our purposes a surface based on a BPR pattern is determined as a set of rectangular grooves (of binary height levels) with grooves and peaks corresponding to values of 1 and 0 in the BPR sequence or array. As such, the PSD from a BPR grating or array is a result of the groove distribution and is not particularly sensitive to the groove shape or roughness of the groove surfaces, top and bottom. As shown in the present work, it is easy to account for any possible affect of fabrication imperfections on the spectral properties of the BPR test surface.

The suggested method involving BPR test surfaces can be adapted for a large variety of profiling instruments including interferometers, interferometric microscopes, atomic force and scanning electron microscopes ${ }^{23}$ and scatterometers. ${ }^{24}$ The listed advantages make BPR based test surfaces ideal for developing international MTF calibration standards.

The utility of using 1D BPR gratings for MTF calibration of the Micromap-570 ${ }^{\mathrm{TM}}$ interferometric microscope has been demonstrated in Refs. $[16,17]$. These surfaces, however, are limited to measuring the MTF along a single direction at a time. BPRAs possess similar properties to the BPRGs with the added advantage of allowing for the direct measurement of the 2D MTF.

\section{CHARACTERIZATION OF PROTOTYPE BPRA'S}

\subsection{Fabrication of BPRA prototypes}

A set of nine prototype BPRA samples was fabricated using high-resolution electron beam lithography and Induction Coupled Plasma (ICP) etching into a silicon substrate. The datasets representing the pseudo-random patterns are generated according the formulae presented in Sec. 2.1. The same dataset is used for all of the prototypes and consists of $4021 \times 4019$ elements. A value of 1 in the data set corresponds to rectangular groove in the substrate and values of 0 represent peaks, or portions that are not etched. The nine prototypes can be thought of as three sets of three BPRAs, with each set etched to a different depth: $49 \mathrm{~nm}, 63 \mathrm{~nm}$, and $122 \mathrm{~nm}$. Although, in principle, etch depth should only correspond to the overall amplitude of the PSD, in a real measurement setup the etch depth may be an important factor that allows for suppression of some effects due to fabrication imperfections. Having samples etched to different depths allows one to draw a conclusion about potential effects of the imperfections. Additional information regarding the fabrication of these BPRA samples can be found in Ref. [25]. 
Within a set of BPRAs etched to the same depth, each BPRA is different only in regards to fundamental element size which ranges from $200 \mathrm{~nm}$, to $400 \mathrm{~nm}$, to $600 \mathrm{~nm}$. The fundamental element size effectively determines the Nyquist frequency of the surface, which is equal to $1 / 2 \Delta$, where $\Delta$ is the fundamental element size. This is the frequency up to which the PSD is expected to be flat. The lower bound is determined by the total size of the surface which is equal to the product of the fundamental element size and the number of columns/rows in the BPRA. If the BPRA is measured outside of this frequency range, the PSD of the surface is no longer guaranteed to be frequency independent. Thus, a BPRA test surface should fill the entire field of view of the instrument in question, but should not be sampled at rates higher than the Nyquist frequency of the surface.

For the present work the instrument in question is the Micromap ${ }^{\mathrm{TM}}-570$ which can be equipped with five objectives, $2.5 \times, 5 \times, 10 \times, 20 \times$, and $50 \times$. The $640 \times 480$ pixel imaging CCD has an effective pixel size determined by the objective in place: $3.92 \mu \mathrm{m}, 1.96 \mu \mathrm{m}, 0.98 \mu \mathrm{m}, 0.49 \mu \mathrm{m}$ and $0.192 \mu \mathrm{m}$. Thus, in principle the fabricated samples provide suitable surfaces for full aperture MTF measurement of the instrument equipped with all objectives.

\subsection{General consideration of fabrication imperfections}

As was mentioned in Sec. 2.2, the special mathematical properties of a BPRA are such that the PSD inherent to the test surface will have a low sensitivity to groove shape distortion. However, in order to use Eq. (1) for an effective and precise MTF measurement, the PSD inherent to the test surface should be well known a priori. Consequently, it's important to consider how fabrication imperfections, particularly groove shape, will affect the inherent PSD of a given test surface.

Measurements made with the Dimension-3000 Scanning Probe Microscope (SPM) provide critical information on the surface characteristics of the BPRA samples. These measurements provide the basis for developing the theoretical models needed for the MTF calibration work. That is, the SPM data is used to estimate the groove shape distortion by means of curve fitting to a simple smoothing function with a small number of parameters. The model with the parameters found in the curve fitting is then used to calculate the inherent PSD of the surface. This process is discussed in greater detail in a later section.

Each of the nine BPRAs was measured under several different magnifications with the SPM. A three dimensional height distribution obtained from a typical measurement shown in Fig. 1a, shows a $5 \mu \mathrm{m} \times 5 \mu \mathrm{m}$ section of the $400 \mathrm{~nm}$ BPRA etched to $63 \mathrm{~nm}$ drawn to scale. This figure clearly reveals non-ideal sidewall profiles. In other words, the etching did not produce the desirable 90 degree sidewall slopes at the groove boundaries. Figure $1 \mathrm{~b}$ shows a $5 \mu \mathrm{m} \times 5 \mu \mathrm{m}$ array plot of the $600 \mathrm{~nm}$ BPRA etched to $63 \mathrm{~nm}$ with darker shaded regions corresponding to grooves in the substrate. This figure reveals a degree of success for the fabrication in that the shapes of the BPRA elements in the plane of the grating are highly rectangular. Consequently, the major perturbation to the inherent PSD of the test surface will be a result of nonideal (rounded) sidewalls.

In order to understand the affect of rounded sidewalls on the inherent PSD of a BPRA, we start with a theoretical consideration. A smoothing function can be applied to an "ideal" BPRA model to account for rounded sidewalls that mimic a "real" fabricated BPRA surface. A number of different smoothing functions can be used and the following is one example of such a function:

$$
C \boldsymbol{\eta}) A \sum_{-p}^{p} B\left(n+p \mathcal{e}^{-p^{2} / 2 \sigma^{2}},\right.
$$

where $B($ is the original height of the ideal profile at point $m$ in the sequence, $p$ is the number of points on either side of $B\left(r_{-}\right.$to be used in smoothing, $C C_{-}$is the new height of the rounded profile at point $m, A$ is a normalization constant, and $\sigma^{2}$ determines the 'strength' of the smoothing function. The free parameters $\sigma^{2}$ and $p$ can be adjusted to vary the shape distortion from very slight rounding to relatively large distortions. Figure 2a shows 1D height profile traces of the first few elements from several BPRA models in which only the strength of rounding has been varied. These models were created using a fundamental element size of 0.6 units, which corresponds to a Nyquist frequency of $\sim 0.8$ units $^{-1}$. The calculated PSD's from these models are shown in Fig. 2a. Although the data presented in Fig. 2 show only a small portion of the numerical models created and analyzed, the results are typical. 

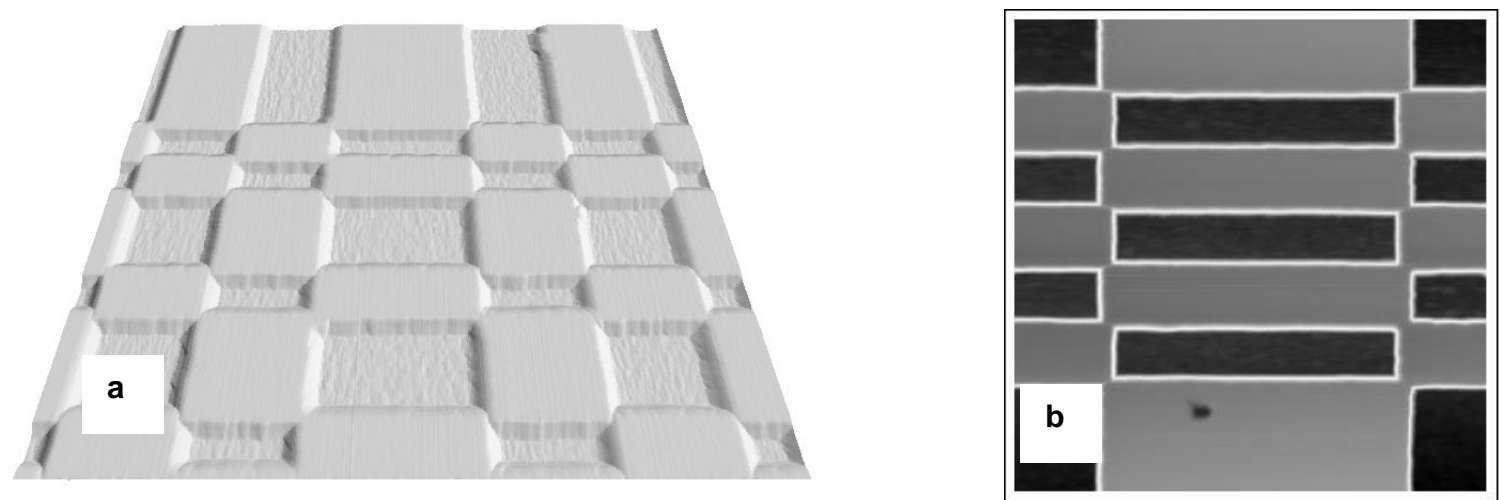

Fig. 1: 3D height distribution of a $5 \mu \mathrm{m} \times 5 \mu \mathrm{m}$ section of the $400 \mathrm{~nm}$ BPRA, etch depth $63 \mathrm{~nm}$, as measured with the SPM, (a). 2D height distribution array plot of $5 \mu \mathrm{m} \times 5 \mu \mathrm{m}$ section of the $600 \mathrm{~nm}$ BPRA, etch depth $63 \mathrm{~nm}$, as measured with the SPM, (b). Dark shaded regions represent grooves in the substrate.
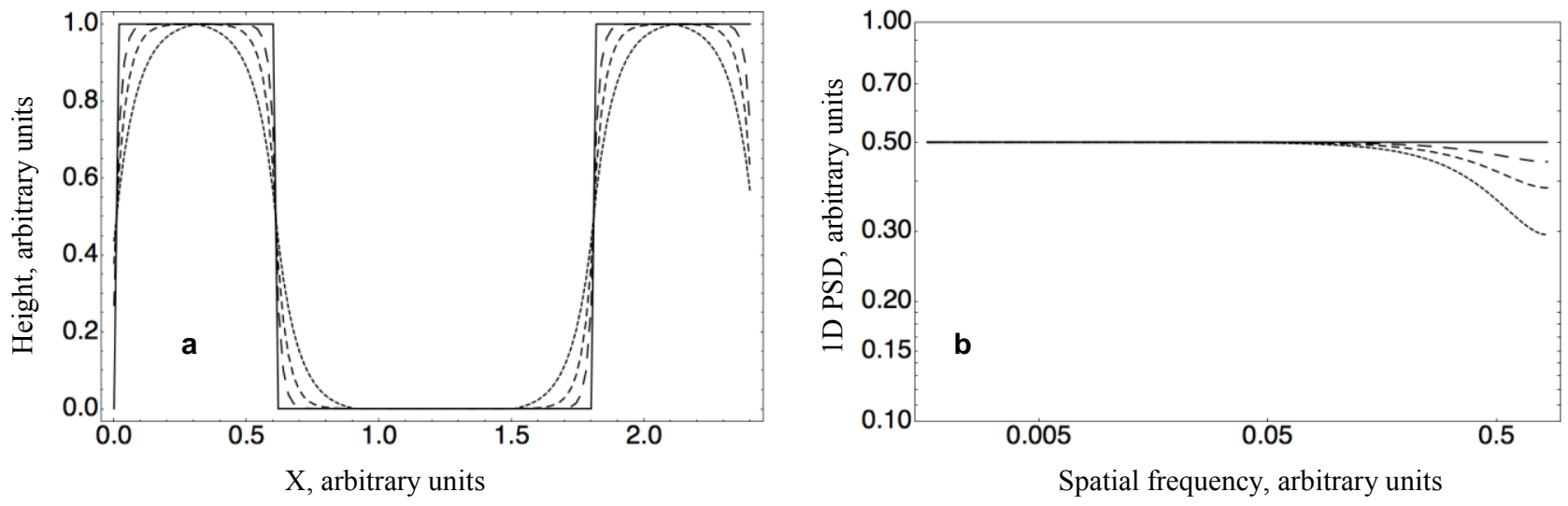

Fig. 2: One dimensional height profiles of the first few elements of model BPRA's with different degrees of rounding, (a). The rounded edges approximate fabrication imperfections of "real" BPRA's. Calculated 1D PSD's are shown in (b). The curves with increasing deviations from flat at the higher spatial frequencies correspond the models with increasing rounding.

\subsection{Micromap ${ }^{\mathrm{TM}}-\mathbf{5 7 0}$ measurements}

In general, it cannot be expected that an entire BPRA fits perfectly within the field of view of the microscope. In most cases only a portion of the BPRA falls in the field of view. It was shown in Ref. [17] that averaging the PSD spectra of multiple measurements, obtained by successively shifting the field of view to different portions of a SUT, accurately recovers the PSD spectrum of the surface. Additionally, averaging several measurements significantly reduces the speckle noise. Of course, the range of spatial frequencies in the measured PSD spectrum is defined by the total size of the field of view in the lower end and by the effective pixel size in the higher end. It is important to make the distinction between the detectable spatial frequency range and the spatial frequency range used to describe the BPRA. For the BPRA, the spatial frequency range is determined as the range over which the PSD is invariant. The detectable range will generally be just a portion of the BPRA frequency range, being cutoff in the low frequency range by the size of the detector and in the high frequency range by the effective pixel size.

Each of the nine BPRA's was measured with Micromap ${ }^{\mathrm{TM}}-570$ equipped with all five objectives. Using PSD software developed at the ALS OML, ${ }^{5-7}$ the 2D PSD is calculated for each surface. The software includes the option to average the PSD's from several different measurements. For the ease of visualization, the 2D PSD's have been integrated along the $\mathrm{x}$ and $\mathrm{y}$ directions to produce two 1D PSD's along the $\mathrm{y}$ and $\mathrm{x}$ directions, respectively. The results of these PSD calculations are shown for $400 \mathrm{~nm}$ BPRA with the $122 \mathrm{~nm}$ etch depth and the $600 \mathrm{~nm}$ BPRA with the $49 \mathrm{~nm}$ etch depth, Figs. 3,4 . 
The PSD's exhibit a relatively high degree of flatness at the lowest spatial frequencies for each given objective. As the frequency increases, however, there is a rather quick and steep roll off of the PSD of several orders of magnitude. This data clearly demonstrates the major impact of the instrumental MTF and the need to calibrate this effect. Only at a small number of the lowest spatial frequencies for a given objective do the PSD's appear to be unaffected by the instrumental MTF.

The PSD's in Fig. 4 obtained from a shallower etched BPRA do not exhibit the same degree of flatness in the low spatial frequency ranges as those in Fig. 3. As was noted in Sec. 3.1, a difference in etch depth should really only manifest itself in the PSD as an overall shift of the PSD amplitude. These raised low frequency tails start to flatten out as the magnification is increased. Upon examining the height distribution data obtained directly from Micromap ${ }^{\mathrm{TM}}-570$ measurements, the source of these perturbations becomes clear.
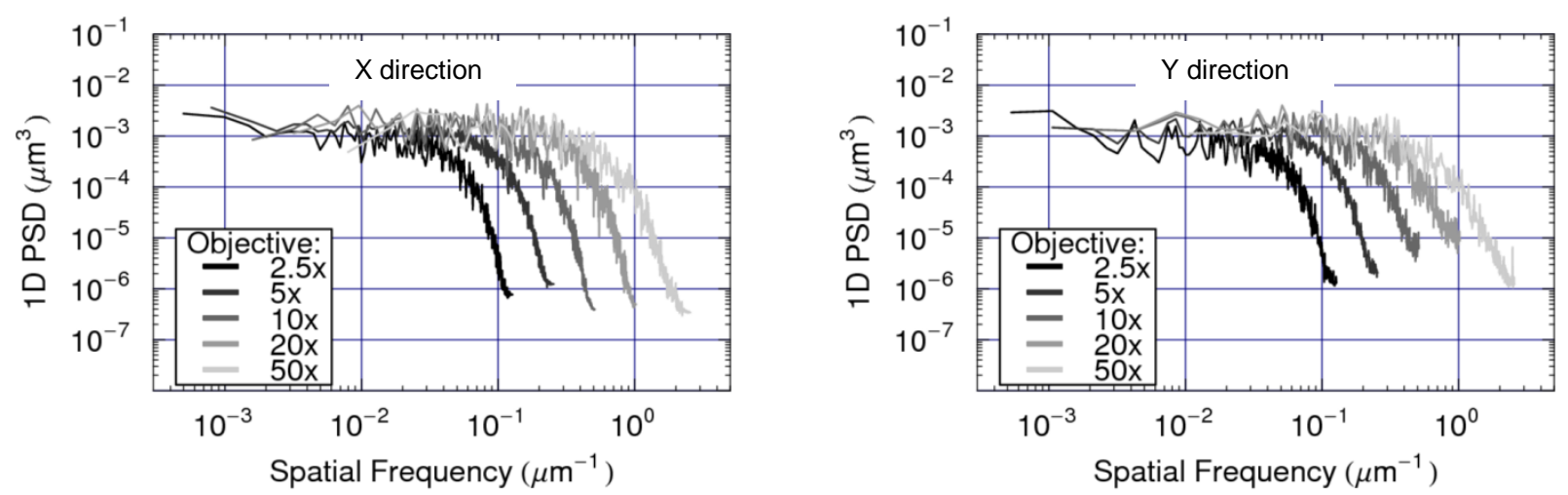

Fig. 3: 1D PSD's obtained by integration of measured 2D PSD of $400 \mathrm{~nm}$ BPRA etched to $122 \mathrm{~nm}$. The PSD's exhibit a high degree of flatness in the low spatial frequency range, but the steep high frequency roll off clearly demonstrates the need to calibrate the MTF effect.
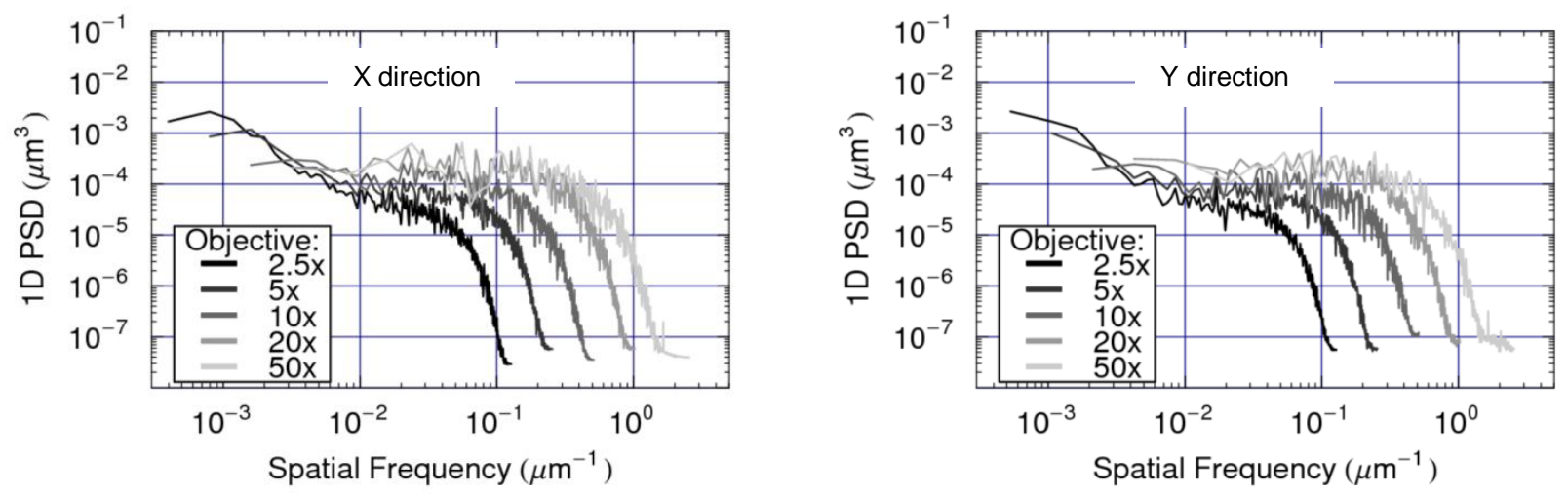

Fig. 4: 1D PSD's obtained by integration of measured 2D PSD of $600 \mathrm{~nm}$ BPRA etched to $43 \mathrm{~nm}$. The raised low frequency tails clearly visible PSD's from the $2.5 \times$ and $5 \times$ objectives indicate the contribution of low frequency surface variations of the cheap silicon wafer into which the BPRA's are etched. For the higher magnification objectives, these contributions are not noticed and the PSD's are relatively flat in the low spatial frequency ranges.

Figure 5 shows height distribution measurements of the silicon substrate next to one of the samples and of the $600 \mathrm{~nm}$ BPRA with $43 \mathrm{~nm}$ etch depth. The measurements were made with Micromap $^{\mathrm{TM}}-570$ when equipped with the $5 \times$ objective. The measurement of the silicon substrate, Fig. 5a, clearly reveals low frequency surface variations in the silicon substrate with peak to valley values on the order of $8 \mathrm{~nm}$. When examining the height distribution of the BPRA, Fig. 5b, along with the BPR pattern, the surface variations of the silicon substrate also show through. Evidently, when the etch depth is not sufficiently deep, the inherent waviness of the silicon wafer distorts the measured PSD's, particularly in the low frequency range. This effect is not noticed when measuring with higher magnification objectives as these frequency contributions are cut off. 
Because similar raised low frequency tails are not noticeable in Fig. 3, it is possible to conclude that etch depths of 122 $\mathrm{nm}$ are deep enough to suppress perturbations due the silicon substrate's surface variations. For future samples, a better approach than setting limits on etch depth would be to start with higher quality polished substrates.
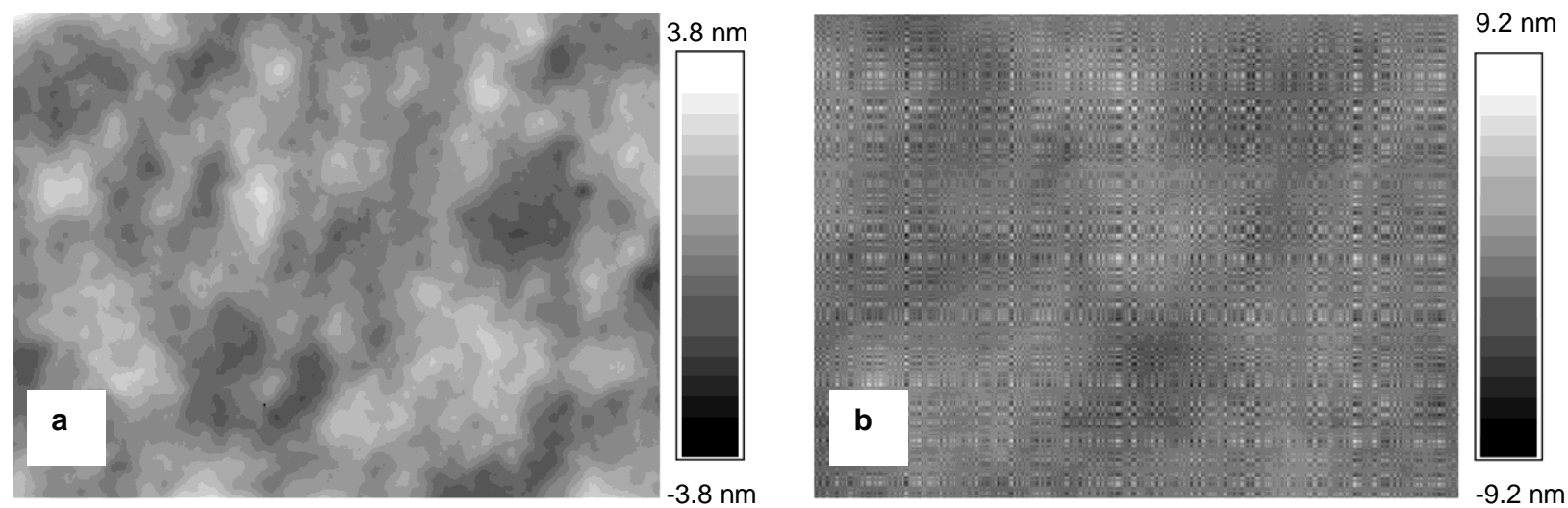

Fig. 5: Micromap ${ }^{\mathrm{TM}}-570$ measurements with the $5 \times$ objective of the bare silicon surface (a) and shallow $49 \mathrm{~nm}$ etch depth $600 \mathrm{~nm}$ BPRA (b). The large-scale roughness of a standard silicon wafer is evident at lower spatial frequencies. The low frequency roughness limits the use of these reference samples in calibrating the $2.5 \times$ and $5 \times$ objectives. Polished silicon flats should not have this roughness.

\section{AB INITIO MTF CALIBRATION}

\subsection{BPRA modeling}

The process of calibrating the MTF of the Micromap ${ }^{\mathrm{TM}}-570$ necessarily begins by estimating the perturbation to the inherent PSDs of the BPRAs due to groove shape distortion resulting from the fabrication process. As mentioned in Sec. 3.3, this is completed by means of curve fitting SPM data. Of the three sets of BPRAs, the samples etched to $63 \mathrm{~nm}$ yielded highest quality SPM data. That is, the height traces exhibit the smoothest and most consistent height distributions, which allows for the most accurate modeling. For this reason, the $200 \mathrm{~nm}, 400 \mathrm{~nm}$, and $600 \mathrm{~nm}$ BPRAs etched to $63 \mathrm{~nm}$ were chosen for an ab initio approach to the MTF correction.

The fitting procedure used to estimate the groove shape begins by taking a trace of the height profile of a single peak to groove boundary from the SPM data, see Fig. 6a. The first step is to create a new dataset that is an "ideal" representation of the real BPRA sample. This can be accomplished by forcing the SPM height profile to binary height levels using a threshold height. Above the threshold height the "ideal" dataset will have a value equal to the average peak height, below the threshold the value will be 0, see Fig 6b. A smoothing function is then applied to the "ideal" dataset that approximates the rounding. The function used here is the same as Eq. (4). The free parameters $\sigma^{2}$ and $p$ are varied and the best fit parameters are determined by minimization of mean square root deviation, see Fig. 6c. Using different height profiles from different portions of the SPM data, the same fitting procedure is repeated. The final best fit parameters are found as the average of the best fit parameters found in the individual fitting routines. In this way best fit parameters were calculated from the SPM data obtained from the $600 \mathrm{~nm}$ BPRA etched to $63 \mathrm{~nm}$.

There is a caveat regarding these parameters for creating a model BPRA. A $5 \mu \mathrm{m} \times 5 \mu \mathrm{m}$ SPM scan of the $600 \mathrm{~nm}$ BPRA will encompass about eight BPRA elements in each direction. For a typical SPM scan, which consists of $512 \times$ 512 data points, a single BPRA element corresponds to $\sim 60 \times 60$ data points. Consequently, in order to develop a full scale model BPRA with the best fit parameters, each BPRA element in the ideal model should be subdivided into $\sim 60 \times$ 60 elements before the rounding can be applied. However, even for a relatively small BPRA of $\sim 100 \times 100$ elements, these subdivisions cause the datasets to grow huge and become rather cumbersome for calculations. To circumvent this issue a new set of parameters derived using significantly fewer subdivisions $(\sim 7)$ that approximate the curve found using the $\sim 60$ subdivision curve. As a check of the validity of this approximation, the 60 and 7 subdivision rounding parameters were applied to a 1D BPR sequence. The 1D PSD's of these two sequences was is compared and found to be nearly identical. 
Another method for reducing the size of the model datasets is to use smaller BPRA's (i.e. fewer elements, $\sim 2000 \times$ 2000). It has been shown that fabrication imperfections of type presented here distort only the high frequency range of the PSD. In contrast, reducing the total size of the BPRA only affects the low spatial frequencies in that they are simply cut off. As such, a rounded model BPRA with a smaller number of elements will still provide the necessary information on the high frequency perturbations of the PSD due to the rounding. Moreover, small BPRA's require far less time to model, evaluate and optimize.
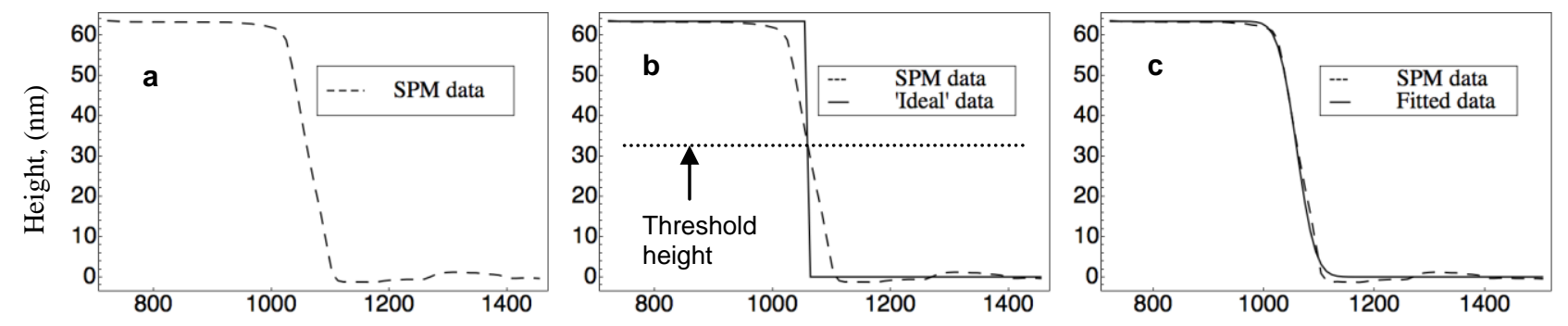

Fig. 6: Viewgraphs illustrating the different steps in the curve fitting procedure used to develop a best fit model of the real BPRA sample. A height profile from the SPM data of a single peak to groove boundary, (a). A dataset representing an "ideal" BPRA is created by forcing the SPM to binary height levels using a threshold height, (b). A smoothing function, like that of Eq. (4) is applied to the "ideal" dataset to approximate the rounding of the real BPRA sample, (c).

A model BPRA was created based on the above prescriptions and the corresponding 2D PSD inherent to the particular realization of the BPRA sample was calculated. It was found that the inherent PSD is expected to deviate from a flat PSD by $\sim 15 \%$ at the Nyquist frequency of the BPRA. In the detectable range of the measurement system, as determined by the objective, the deviation from a flat PSD can be considerably less. For example, the Nyquist spatial frequency of a $600 \mathrm{~nm}$ BPRA is $0.833 \mu^{-1}$. The highest detectable spatial frequency of the Micromap ${ }^{\mathrm{TM}}-570$ when equipped with the $10 \times$ objective (effective pixel size $0.98 \mu \mathrm{m}$ ) is $\sim 0.5 \mu \mathrm{m}^{-1}$. At this spatial frequency, the deviation from a flat PSD is only about $8 \%$.

\subsection{MTF calibration of Micromap ${ }^{\mathrm{TM}}-570$ interferometric microscope}

For an $a b$ initio calibration, we use the MTF that is directly found as the square root of the ratio of the measured 2D PSD to the 'theoretical' 2D PSD, which is calculated using the BPRA model of the $600 \mathrm{~nm}$ BPRA etched to $63 \mathrm{~nm}$. In order to relate the PSD measured by the Micromap ${ }^{\mathrm{TM}}-570$ to the PSD calculated from the theoretical model, additional considerations are needed. The PSD's from the BPRA models presented up to this point correspond to measuring a real BPRA in which the detector has the exact same number of pixels as the BPRA has elements and in which the detector is aligned such that each pixel perfectly maps to a single element of the BPRA. Such a system would be an ideal measuring system. This is essentially an impossible scenario in a real lab setting. The Micromap ${ }^{\mathrm{TM}}-570$ detector has $640 \times 480$ pixels, with an effective pixel size that varies with the objective being used. This results in two differences from the ideal scenario. First, the entire field of view, for the majority of the cases, will be smaller than the BPRA. Second, for BPRA's presented in this work, a single pixel in the detector may 'see' just a portion of, or more than, one element of the BPRA.

Accordingly, the theoretical PSD is calculated in a way that reflects how real measurements are made. To account for the effective pixel size of the detector, we apply a simple convolution procedure. For example, for the $10 \times$ objective, the effective pixel size of the detector is $0.98 \mu \mathrm{m} \times 0.98 \mu \mathrm{m}$. We convolve the model by calculating the average height for each adjacent section of $0.98 \mu \mathrm{m} \times 0.98 \mu \mathrm{m}$ of the model. After the convolution, several $640 \times 480$ sections are extracted, which is effectively equivalent to taking several measurements with the Micromap ${ }^{\mathrm{TM}}-570$ equipped with the $10 \times$ objective. The 2D PSD is then calculated by averaging the PSD's of the individual sections. Averaging several PSD's from different portions of the surface has been shown to be an effective method to reduce the amount of noise in the PSD.

In order to find the MTF we take the square root of the ratio of the measured PSD to the 'theoretical' PSD. This is a fairly straightforward calculation, but there is an intermediary step. It is well known that the MTF will not affect the PSD at the lowest frequencies. In other words, the MTF should be equal to one at the lowest spatial frequencies. In order to ensure this requirement is met, the 'theoretical' 2D PSD must be normalized to match the amplitude of the measured PSD at the lowest frequencies. Determining the value of this amplitude, however, is not trivial. 
The goal is to use the value of the measured 2D PSD at the low spatial frequencies to normalize the theoretical PSD such that the MTF is unity at the lowest spatial frequencies. Using a single value of the measured PSD at the lowest frequency is problematic since a single data point is particularly influenced by random noise. A better approach is to average the measured PSD over a range of frequencies to reduce random noise effects. On the other hand, as the frequency range increases, the MTF starts to distort the measured PSD, which will adversely affect the normalization of the theoretical PSD. So, in order to determine an accurate value for the amplitude of the measured PSD for normalization of the theoretical PSD, the frequency range over which to average the measured PSD should be large enough to average out random noise effects, but not too large as to incorporate the MTF distortions at the higher frequencies. Since the definition of this frequency range is somewhat arbitrary, it is important to examine the effects of using different frequency ranges in determining the theoretical 2D PSD amplitude.

To check this, the measured 2D PSD was averaged over varying frequency ranges. A suitable frequency range must include the lowest frequencies but exclude the zero frequency and be large enough to suppress contributions of noise. By varying the frequency range from a $5 \times 5$ matrix of the lowest frequencies to an $8 \times 8$ matrix, the average value of the PSD was calculated. For measurements made with the $10 \times, 20 \times$ and $50 \times$ objectives, it was found that the average value of the PSD changes by only $\sim 5-7 \%$. Consequently, only an error of about $5-7 \%$ is expected when normalizing the theoretical PSD. For the $5 \times$ and $2.5 \times$ objectives, the variation is much larger. This is due to the larger field view which encompasses more low frequency surface variations of the silicon substrate. Thus, the BPRAs etched to $63 \mathrm{~nm}$ are not particularly suitable for MTF calibration of the $5 \times$ and $2.5 \times$ objectives.

Using the procedure outlined above, the MTF has been experimentally determined for the Micromap ${ }^{\mathrm{TM}}-570$ equipped with the $10 \times$ objective. The MTF found in this way was then used to correct measurements of the other BPRA's ( $200 \mathrm{~nm}$ and $400 \mathrm{~nm}$ ) with the same etch depth, Fig. 7. The lower curves are the un-corrected curves and the top curves have the MTF correction applied. These results offer a good indication of success. The PSDs inherent to these BPRAs is expected to be largely flat across the entire detectable spatial frequency range. The corrected PSDs clearly demonstrate that the when the experimentally determined instrumental MTF has been corrected, the recovered PSD exhibits precisely the expected characteristics. Note that a roll off of more than three orders of magnitude in the high frequency range of the PSDs has been corrected when the MTF calibration is applied.
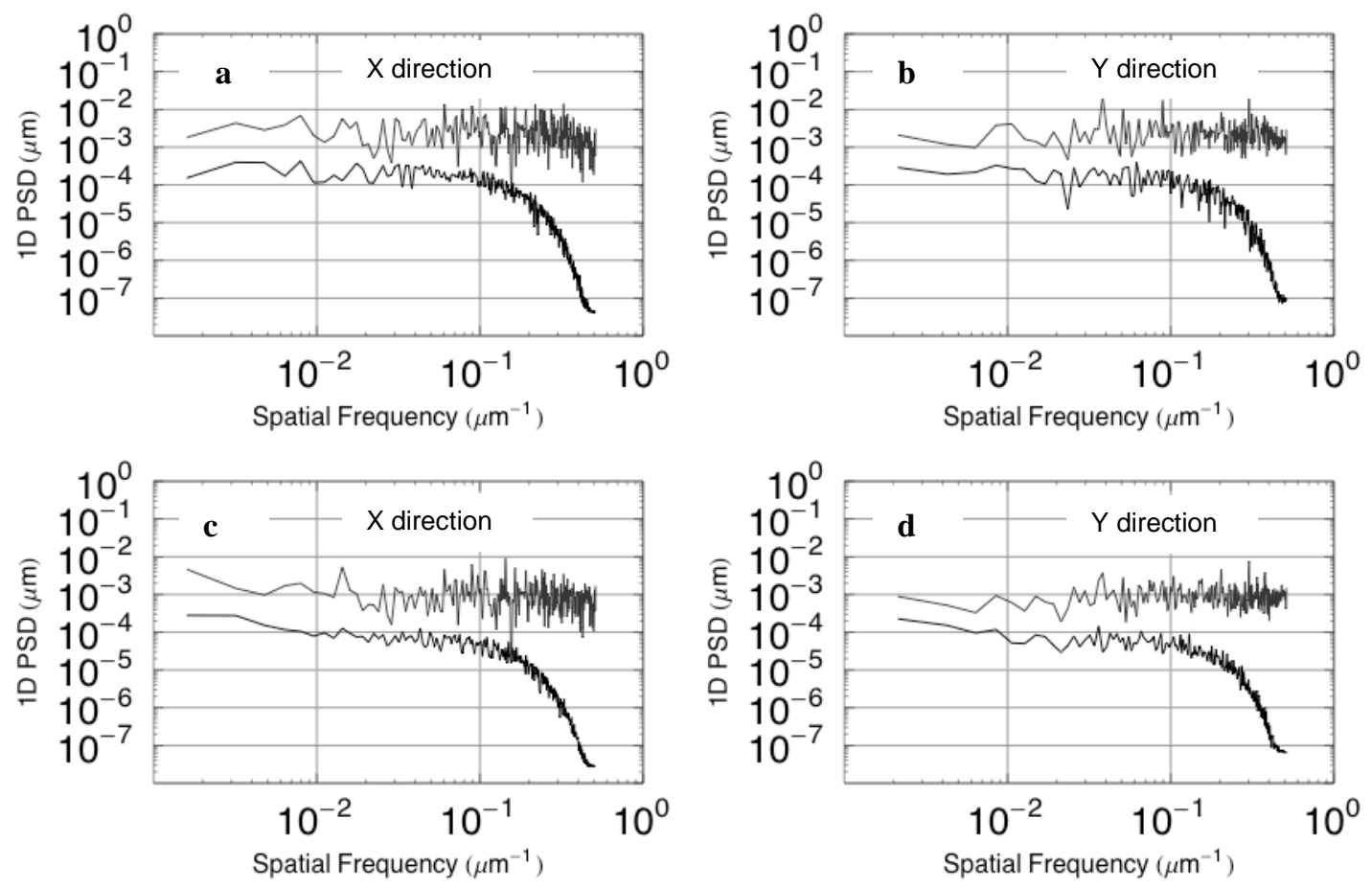

Fig. 7: 1D PSD measurements along the $\mathrm{x}$, (a) and (c), and y, (b) and (d), directions of the BPRA with $400 \mathrm{~nm}$, (a) and (b), and the $200 \mathrm{~nm}$, (c) and (d), elementary step (bottom curves) along with PSD's obtained after applying experimentally determined MTF correction (top curves). Measurements were made with 10× objective. 
An important note is that the amplitude offsets seen between the un-corrected and corrected PSD's in Fig. 7 are not manufactured. The MTF correction is applied to the two dimensional PSD of a BPR test surface that is inherently frequency independent. In this case, convolution of the corrected 2D PSD distribution to a 1D PSD spectrum in the $x$ direction, involving summing over all frequencies in the y direction, will affect the 1D PSD at low $\mathrm{x}$-frequencies due to the correction at higher $y$-frequencies. For this reason one should expect a significant change of the roughness number after the MTF correction has been applied, particularly for surfaces with random distributions of surface height. For optics, which generally have a fractal like 1D PSD that decreases sharply with increasing spatial frequency, ${ }^{26}$ the PSD distribution at high $\mathrm{y}$-frequencies does not significantly change the low frequency part of the 1D PSD in $\mathrm{x}$-direction.

\section{CONCLUSION}

Test surfaces suitable for the calibration of the two dimensional MTF of surface profilometers have been fabricated based on binary pseudo-random arrays. The frequency response of an ideal profilometer measuring an ideal BPRA should be characterized by a perfectly flat white-noise-like response. As such, BPRAs provide direct approach to measuring the 2D MTF of an instrumental system and provide uniform sensitivity at all spatial frequencies.

Special considerations have been made to account for real measuring systems and imperfect BPRAs. It has been shown that for BPRAs with less than ideal groove shape, it is possible to approximate the perturbation to the inherent PSD by means of curve fitting SPM data of the SUT. The inclusion of fabrication imperfections in BPRA models is generally characterized by a high frequency roll off in the PSD with little to no effect at lower spatial frequencies. Because the range of detectable spatial frequencies is limited by the measuring system, much of the effect of fabrication imperfections is not detectable. Therefore, rough estimates of the fabrication imperfection of a real BPRA suffice to account for $<\% 10$ deviations from a flat PSD in the detectable range.

As an example, results of measuring the MTF of the Micromap ${ }^{\mathrm{TM}}-570$ equipped with the $10 \times$ objective using the $600 \mathrm{~nm}$ BPRA etched to $63 \mathrm{~nm}$ as a reference surface have been presented. Using the experimentally determined MTF, measurements of the other BRPA's (200 nm, $400 \mathrm{~nm}$ ) have been corrected. The corrected PSD's exhibit precisely the characteristics that are expected for such surfaces, providing conclusive evidence of the applicability of this MTF calibration procedure. Similar results have been obtained for the $20 \times$ and $50 \times$ objectives.

It was found that surface variations of the silicon wafer into which the samples are etched, makes it difficult to use this procedure for MTF measurement of the $2.5 \times$ and $5 \times$ objectives. The samples etched $122 \mathrm{~nm}$ seem to suppress this negative influence. However, it appears more difficult to characterize fabrication imperfections of these samples from the SPM data, due either to a bad SPM tip or real imperfections in the BPRA's.

A considerably simpler approach to this MTF calibration procedure would begin with BPRAs with square, or very nearly square, edges. In such a case, the imperfections of the BPRA elements are so slight that the effect on the PSD is negligible. Consequently, BPRAs with square features would permit us to skip the process of developing a best fit model of the real BPRAs and estimating the distortion of the PSD. Rather, we can assume that since the BPRAs are essentially ideal, that the PSD will be perfectly flat. A number of different fabrication techniques are under preliminary testing with positive results. Once the best fabrication technique has been chosen, new sets of BPRAs will be etched into a super polished silicon substrate, which will allow the calibration of instruments with larger fields of view.

\section{ACKNOWLEDGEMENTS}

The authors are grateful Howard Padmore for extremely useful discussions. The Advanced Light Source is supported by the Director, Office of Science, Office of Basic Energy Sciences, Material Science Division, of the U.S. Department of Energy under Contract No. DE-AC02-05CH11231 at Lawrence Berkeley National Laboratory. 


\section{DISCLAIMER}

This document was prepared as an account of work sponsored by the United States Government. While this document is believed to contain correct information, neither the United States Government nor any agency thereof, nor The Regents of the University of California, nor any of their employees, makes any warranty, express or implied, or assumes any legal responsibility for the accuracy, completeness, or usefulness of any information, apparatus, product, or process

disclosed, or represents that its use would not infringe privately owned rights. Reference herein to any specific commercial product, process, or service by its trade name, trademark, manufacturer, or otherwise, does not necessarily constitute or imply its endorsement, recommendation, or favoring by the United States Government or any agency thereof, or The Regents of the University of California. The views and opinions of authors expressed herein do not necessarily state or reflect those of the United States Government or any agency thereof or The Regents of the University of California.

\section{REFERENCES}

[1] Assoufid, L., Hignette, O., Howells, M., Irick, S. C., Lammert, H. and Takacs, P. Z., "Future metrology needs for synchrotron radiation grazing-incidence optics," Nucl. Instrum. and Meth. in Phys. Research A 467-468, 267-70 (2001).

[2] Takacs, P. Z., [X-Ray Mirror Metrology], in Handbook of Optics, 3rd edition, M. Bass, ed., vol. V, ch. 46 (in press).

[3] Boreman, G. D., [Modulation Transfer Function in Optical and Electro-optical Systems], SPIE Press, Bellingham, 1 (2001).

[4] Bracewell, R. N., [The Fourier Transform and Its Applications], McGraw-Hill Publishing Company, New York, 1 (1986).

[5] Yashchuk, V. V., Franck, A. D., Irick, S. C., Howells, M. R., MacDowell, A. A. and McKinney, W. R., "Two dimensional power spectral density measurements of x-ray optics with the Micromap interferometric microscope," Proc. of SPIE 5858, pp. 58580A-1-12 (2005).

[6] Yashchuk, V. V., Irick, S. C., Gullikson, E. M., Howells, M. R., MacDowell, A. A., McKinney, W. R., Salmassi, F. and Warwick, T., "Cross-check of different techniques for two dimensional power spectral density measurements of x-ray optics," Proc. of SPIE 5921, pp. 59210G-1-12 (2005).

[7] Yashchuk, V. V., Gullikson, E. M., Howells, M. R., Irick, S. C., MacDowell, A. A., McKinney, W. R., Salmassi, F., Warwick, T., Metz, J. P. and Tonnessen, T. W., "Surface roughness of stainless-steel mirrors for focusing soft $\mathrm{x}$ rays," Appl. Opt. 45(20), 4833-42 (2006).

[8] Church, E. L., Jenkinson, H. A. and Zavada, J. M., "Relationship between surface scattering and microtopographic features," Opt. Eng. 18(2), 125-36 (1979).

[9] Stover, J. C., [Optical Scattering], second edition, SPIE Optical Engineering Press, Bellingham, 1 (1995).

[10] Attwood, D., [Soft X-rays and Extreme Ultraviolet Radiation], Cambridge University Press, New York, 1 (1999).

[11] Boreman G. D. and Yang, S., "Modulation transfer function measurement using three- and four-bar targets," Appl. Opt. 34, pp. 8050-8052 (1995).

[12] Barakat, R., "Determination of the optical transfer function directly from the edge spread function," JOSA 55, 1217 (1965).

[13] Tatian, B., "Method for obtaining the transfer function from the edge response function," JOSA 55, 1014 (1965).

[14] Takacs, P. Z., Li, M. X., Furenlid, K. and Church, E. L., "Step-height standard for surface-profiler calibration," Proc. of SPIE 1995, 235-44 (1993).

[15] Daniels, A., Boreman, G. D., Ducharme, A. and Sapir, E., "Random transparency targets for modulation transfer function measurement in the visible and IR," Opt. Eng. 34, 860-868 (1995).

[16] Yashchuk, V. V., McKinney, W. R. and Takacs, P. Z., "Binary Pseudorandom Grating Standard for Calibration of Surface Profilometers," Opt. Eng. 47(7), 073602-1-5 (2008).

[17] Yashchuk, V. V., McKinney, W. R. and Takacs, P. Z., "Binary Pseudorandom Grating as a Standard Test Surface for measurement of Modulation Transfer Function of Interferometric Microscopes," Proc. SPIE 6704, 670408/1-12 (2007).

[18] Dicke, R. H., "Scatter-hole cameras for X-rays and gamma rays,” Astrophys. J. 153, L101-L106 (1968).

[19] Ables, J. G., "Fourier transform photography: a new method for X-ray astronomy," Proc. Astron. Soc. Aust. 4, 172173 (1968). 
[20] Fenimore, E. E. and Cannon, T. M., “Coded aperture imaging with uniformly redundant arrays,” Appl. Opt. 17(3), 337-47 (1978).

[21] Bardell, P. H., McAnney, W. H. and Savir, J., [Built-in test for VLSI pseudorandom techniques]. John Wiley and Sons Inc., New York, 1 (1987).

[22] Marchesini, S., Boutet, S., Sakdinawat, A. E., Bogan, M. J., Bajt, S., Barty, A., Chapman, H. N., Frank, M., HauRiege, S. P., Szöke, A., Cui, C., Shapiro, D. A., Howells, M. R., Spence, J. C. H., Shaevitz, J. W., Lee, J. Y., Hajdu, J., and Seibert, M. M., "Massively parallel X-ray holography," Nat. Photonics 2, 560-563 (2008).

[23] The corresponding developments and experiments are in progress at the Advanced Light Source (ALS) Optical Metrology Laboratory (OML).

[24] Barber, S. K., Soldate, P., Anderson, E. H., Cambie, R., McKinney, W. R., Takacs, P. Z., Voronov, D. L. and Yashchuk, V. V., "Binary pseudo-random gratings and arrays for calibration of the modulation transfer function of surface profilometers: recent developments", Abstract to $245^{\text {th }}$ PTB Seminar on Scatterometry and Ellipsometry on structured surfaces, Braunschweig, Germany (18-19 March, 2009).

[25] Barber, S. K., Soldate, P., Anderson, E. H., Cambie, R., McKinney, W. R., Takacs, P. Z., Voronov, D. L. and Yashchuk, V. V., "Development of Pseudo-random Binary Arrays for Calibration of Surface Profile Metrology Tools," Abstract to The $53^{\text {rd }}$ International Conference on Electron, Ion, and Photon beam Technology and Nanofabrication, Marco Island, Florida (May 26-28, 2009).

[26] Church, E. L., "Fractal surface finish," Appl. Opt. 27(8), 1518-1526, (1988). 